\title{
The mitochondrial genome of the egg- laying flatworm Aglaiogyrodactylus forficulatus (Platyhelminthes: Monogenoidea)
}

\author{
Lutz Bachmann ${ }^{1 *}$, Bastian Fromm², Luciana Patella de Azambuja ${ }^{3}$ and Walter A. Boeger ${ }^{3}$
}

\begin{abstract}
Background: The rather species-poor oviparous gyrodactylids are restricted to South America. It was suggested that they have a basal position within the otherwise viviparous Gyrodactylidae. Accordingly, it was proposed that the species-rich viviparous gyrodactylids diversified and dispersed from there.

Methods: The mitochondrial genome of Aglaiogyrodactylus forficulatus was bioinformatically assembled from next-generation illumina MiSeq sequencing reads, annotated, and compared to previously published mitochondrial genomes of other monogenoidean flatworm species.

Results: The mitochondrial genome of A. forficulatus consists of 14,371 bp with an average A + T content of $75.12 \%$. All expected 12 protein coding, 22 tRNA, and 2 rRNA genes were identified. Furthermore, there were two repetitive non-coding regions essentially consisting of 88 bp and 233 bp repeats, respectively. Maximum Likelihood analyses placed the mitochondrial genome of A. forficulatus in a well-supported clade together with the viviparous Gyrodactylidae species. The gene order differs in comparison to that of other monogenoidean species, with rearrangements mainly affecting tRNA genes. In comparison to Paragyrodactylus variegatus, four gene order rearrangements, i. e. three transpositions and one complex tandem-duplication-random-loss event, were detected.
\end{abstract}

Conclusion: Mitochondrial genome sequence analyses support a basal position of the oviparous $A$. forficulatus within Gyrodactylidae, and a sister group relationship of the oviparous and viviparous forms.

Keywords: Brazil, Fish parasites, Gene order, Gyrodactylidae, Mitogenomics, Monogenoidea, Neodermata

\section{Background}

Within flatworms (Platyhelminthes) the parasitic Neodermata represent the most derived forms and are, at least when compared to the free-living flatworm lineages, particularly species rich. They include the ectoparasitic Monogenoidea, and the endoparasitic flukes (Trematoda) and tapeworms (Cestoda).

Among the Monogenoidea, Gyrodactylidae have attracted particular attention as some species have been of great concern for humans. For example, Gyrodactylus salaris Malmberg, 1957 has caused significant ecological

\footnotetext{
* Correspondence: bachmann@nhm.uio.no

${ }^{1}$ Natural History Museum, University of Oslo, PO Box 1172, Blindern 0318,

Oslo, Norway

Full list of author information is available at the end of the article
}

and economic damage to wild stocks of Atlantic salmon (Salmo salar) in Norway and Russia as well as in aquaculture [1]. The majority of the species within Gyrodactylidae are hyperviviparous ectoparasites on actinopterygian fish hosts, but there are also some oviparous gyrodactylid lineages. These lineages were originally placed within the Oogyrodactylidae [2], which was later rejected as paraphyletic by Boeger et al. [3], and Oogyrodactylidae were included in Gyrodactylidae based on morphological synapomorphies.

Oviparous gyrodactylids are restricted to South America and occur on freshwater catfishes, mainly on species of the Loricariidae (Siluriformes). Boeger et al. [4] considered the oviparous species a basal lineage within Gyrodactylidae. Taking into account their geographical restriction to 
continental South America they argued that the speciesrich viviparous gyrodactylids diversified and dispersed from there [4]. Accordingly, hyperviviparity and the loss of 'sticky' eggs were interpreted as synapomorphic key innovations of the viviparous Gyrodactylidae.

In comparison to the enormous diversity of the viviparous gyrodactylids the oviparous lineages appear rather species-poor; until today only 23 species in seven genera have been described [5]. Among them is Aglaiogyrodactylus forficulatus Kritsky, Vianna \& Boeger, 2007, the type-species of the genus, that was originally described from the loricarid host Kronichthys lacerta [5].

In the current study we present the first mitochondrial genome of an oviparous gyrodactylid. The molecular characteristics of the mitochondrial genome of $A$. forficulatus are compared with those of other previously described monogenoidean mitochondrial genomes, and the phylogenetic position of oviparous gyrodactylids is addressed.

\section{Methods}

The oviparous $A$. forficulatus was collected from its type-host, the catfish Kronichthys lacerta Nichols, 1919, from Rio Morato, basin of the Garaqueçaba, Paraná, Brazil $\left(25^{\circ} 12^{\prime} 48^{\prime \prime} \mathrm{S}, 48^{\circ} 17^{\prime} 52^{\prime \prime W}\right)$ on 30 October 2013.

About 200 individual parasites were pooled for DNA extraction with the E.Z.N.A. Tissue DNA kit (Omega Bio-Tek) following the Tissue DNA-Spin Protocol provided with the kit. For high-throughput next-generation sequencing (NGS) of the genomic DNA paired-end libraries were prepared, tagged, and analyzed $(29,107,020$ paired 300 bp reads) on an illumina MiSeq (outsourced to GENterprise GENOMICS, Mainz, Germany).

The mitochondrial genome of $A$. forficulatus was reconstructed by assembling the NGS reads using MITObim 1.8 [6] using essentially the default settings of the program, and, in addition, the program's quality trimming option. The COII sequence of $A$. ctenistus (GenBank accession number KF751723; [7]) was used as seed sequence for the assembly.

Annotation of the mitochondrial genes was done using MITOS [8] and DOGMA [9]. In addition, tRNA genes were also identified using tRNAscan-SE 1.21 [10]. For most protein coding genes (PCGs) only the conserved domains were identified by the two annotation programs. Phylogenetic comparisons with already published monogenoidean mitochondrial genomes (Table 1) were, therefore, used to manually complete these genes' annotation, which was assisted by blast searches of GenBank. Codon usage was analyzed using the Sequence Manipulation Suite v2 [11]. The dot-plot approach of YASS [12] was used to identify and visualize the repeat regions. The mitochondrial gene map was drawn using SnapGene 3.0.
Table 1 The mitochondrial genomes of 11 monogenoidean and two cestode species included in this study for comparative analyses

\begin{tabular}{|c|c|c|c|}
\hline Species & $\begin{array}{l}\text { GenBank accession } \\
\text { number }\end{array}$ & $\begin{array}{l}\text { Length } \\
\text { (bp) }\end{array}$ & References \\
\hline \multicolumn{4}{|l|}{ Monogenoidea: Gyrodactylidea } \\
\hline Aglaiogyrodactylus forficulatus & KU679421 & 14,371 & this study \\
\hline Paragyrodactylus variegatus & KM067269 & 14,517 & [13] \\
\hline Gyrodactylus salaris $^{a}$ & NC008815 & 14,790 & [14] \\
\hline Gyrodactylus thymallia & NC009682 & 14,788 & {$[15]$} \\
\hline Gyrodactylus derjavinoides & NC010976 & 14,741 & [16] \\
\hline \multicolumn{4}{|l|}{ Monogenoidea: Capsalidea } \\
\hline Benedenia seriolae & HM222526 & 13,498 & [17] \\
\hline Benedenia hoshinai & EF055880 & 13,554 & [18] \\
\hline Neobenedenia melleni & JQ038228 & 13,270 & [19] \\
\hline \multicolumn{4}{|l|}{ Monogenoidea: Dactylogyridea } \\
\hline Tetrancistrum nebulosi & NC018031 & 13,392 & {$[20]$} \\
\hline \multicolumn{4}{|l|}{ Monogenoidea: Mazocraeidea } \\
\hline Polylabris halichoeres & NC016057 & 15,527 & [21] \\
\hline Microcotyle sebastis & NC009055 & 14,407 & {$[22]$} \\
\hline Pseudochauhanea macrorchis & NC016950 & 15,031 & [23] \\
\hline \multicolumn{4}{|l|}{ Cestoda } \\
\hline Hymenolepis diminuta & AF314223 & 13,900 & [24] \\
\hline Echinococcus oligarthrus & NC009461 & 13,791 & [25] \\
\hline
\end{tabular}

arecently synonymized by [35]

The assembled mitochondrial genome of A. forficulatus was compared to previously published mitochondrial genomes of other monogenoidean species [13-25] that are listed in Table 1. The mitochondrial genomes of the cestodes Echinococcus oligarthrus [24] and Hymenolepis diminuta [25] served as outgroups. All genes were aligned individually using the online version of MAFFT Alignment v7.245 [26]. Subsequently the individual tRNA, rRNA, and PCG alignments were concatenated into one extended alignment, which did not include the non-coding regions of the mitochondrial genes. GBlock v0.91b [27] and GUIDANCE2 [28] were applied to remove ambiguous and unreliable sections from the concatenated MAFFT alignments.

PartitionFinder v.1.1.1_Mac [29] was used to select the best-fit model of molecular evolution for the concatenated alignments using as recommended the program's "raxml" search option and the Bayesian Information Criterion (BIC) for model selection. Maximum Likelihood analyses were carried out in PHYML 3.0 [30] applying the GTR + I + G model and 1,000 bootstrap replicates for all analyses except those for the Cytb and ND3 genes that were performed applying the GTR + G model.

Comparisons of gene orders of the mitochondrial genomes of $A$. forficulatus and the other monogenoidean 
species included in this study were conducted in CREx [31], a program that infers most parsimonious gene rearrangements based on common intervals.

\section{Ethics approval}

All sampling of parasites (on fish) was done under license number 10007 (Instituto Chico Mendes de Conservação da Biodiversidade - ICMBio, Brazil).

\section{Results and discussion}

Next-generation sequencing of the genomic DNA of $A$. forficulatus delivered 29,107,020 million paired 300 bp reads. The initial assembly with the COII sequence of $A$. ctenistus as seed sequence as well as subsequent control assemblies using various $A$. forficulatus seeds delivered identical mitochondrial DNA (mtDNA) consensus sequences. During the assembly of the mtDNA of $A$. forficulatus particular attention was paid to the two repetitive repeat regions (see below) in order to avoid erroneous base-calling due to misassembled short reads; the final sequence was determined only from those reads that also in part covered the respective flanking regions.

The mtDNA of A. forficulatus is $14,371 \mathrm{bp}$ long, and was assembled from 43,334 quality-trimmed reads with an average coverage of $765 \mathrm{x}$. The overall $\mathrm{A}+\mathrm{T}$ content was $75.1 \%$ and the nucleotide composition was A (30.2 \%) C (9.7 \%), G (15.1\%), and T (44.9\%). The base composition of the tRNA and rRNA genes, PCGs, and repeat regions are listed in Additional file 1. The nucleotide sequence of the assembled mtDNA of $A$. forficulatus was deposited in GenBank with the accession number KU679421.

All genes usually described for the mitochondrial genomes of other Monogenoidea (12 protein coding genes, two rRNAs, and 22 tRNAs) were also identified for A. forficulatus (Fig. 1, Table 2). Accordingly, ATP8 lacks also in A. forficulatus. All genes are coded on the same strand.

Protein coding genes (PCGs) For most PCGs the applied annotation programs only detected the conserved domains. This worked best for the COI and Cytb genes, whereas ND2 was not detected at all. Thus, most PCGs were annotated manually through phylogenetic comparisons with reference to other previously published platyhelminth mitochondrial genomes (Table 1). Translation into amino acid sequences was straightforward using the flatworm mitochondrial code [32]. All PCG except for ND2 and ND4 use ATG as start codon. For ND2 and ND4, however, it proved difficult to identify the beginning of the gene, as there was no canonical start codon of flatworm mitochondria (reviewed in [33]) in frame in the respective region. As a working hypothesis, we suggest that both ND2 and ND4 start with ATT, which was reported earlier as a rarely used alternative start codon in other flatworm mitochondria (e.g. [13, 34]). All PCGs terminate with one of the canonical flatworm stop codons TAG or TAA (Table 2). As reported for other monogenoidean mitochondrial genomes, A. forficulatus shows a strong codon bias for PCGs. The most commonly used codons for a particular amino acid in $A$. forficulatus were, however, in all cases the same as for P. variegatus (Additional file 2).

rRNA genes The $12 \mathrm{~S}$ and $16 \mathrm{~S}$ rRNA genes were identified of being $729 \mathrm{bp}$ and $928 \mathrm{bp}$ long, respectively. The A + T content was $77.4 \%$ for the $12 \mathrm{~S}$ gene and for the $75.6 \%$ $16 \mathrm{~S}$ rRNA gene, and thus very similar to the average of the whole mitochondrial genome.

tRNA genes All 22 tRNA genes were identified by MITOS [8] and DOGMA [9] that, however, failed to detect tRNA P for prolin. tRNAscan-SE [10] confirmed eight tRNAs but could not predict the remaining ones (Table 2). The secondary cloverleaf structures of the predicted tRNAs are compiled in Additional file 3. The tRNAs C, S1, and S2 lack the DHU arm.

\section{Genetic diversity and phylogenetic analyses}

Based on the concatenated MAFFT alignments $(15,261$ positions), the mitochondrial genome of the egg laying A. forficulatus was found in a sister-group relationship to the mitochondrial genomes of the other viviparous Gyrodactylidea, i.e. G. salaris, G. thymalli, G. derjavinoides, and P. variegatus (Fig. 2) with high statistical support. However, it has to be taken into account that the depicted ML tree does not provide a comprehensive phylogenetic hypothesis for the Monogenoidea since it is only based on the limited number of available mitochondrial genomes. All included Capsalidea, Mazocraeidea, respectively, also clustered together with high statistical support. Despite the limitations of the analyses the observed phylogenetic affinity of $A$. forficulatus to the included viviparous Gyrodactylidea species makes sense, as the grouping is congruent with the results of earlier morphological analyses [3].

Similar tree topologies with respect to the Gyrodactylidea clade were also obtained when analyzing the concatenated tRNA (bootstrap support 50) genes as well as the COI (100), COII (86), COIII (65), Cytb (77), ND1 (100), ND3 (66), ND4L (93), ND4 (99), and ND5 (100) genes individually. When analyzing the rRNA genes as well as the ATP6, ND2, and ND6 genes individually somewhat different tree topologies with affinities of $A$. forficulatus to other species or clades were obtained. Similar tree topologies were also obtained when using the concatenated GBlock and GUIDANCE2 alignments, respectively (Additional file 4). With the unreliable parts of the original MAFFT alignment 


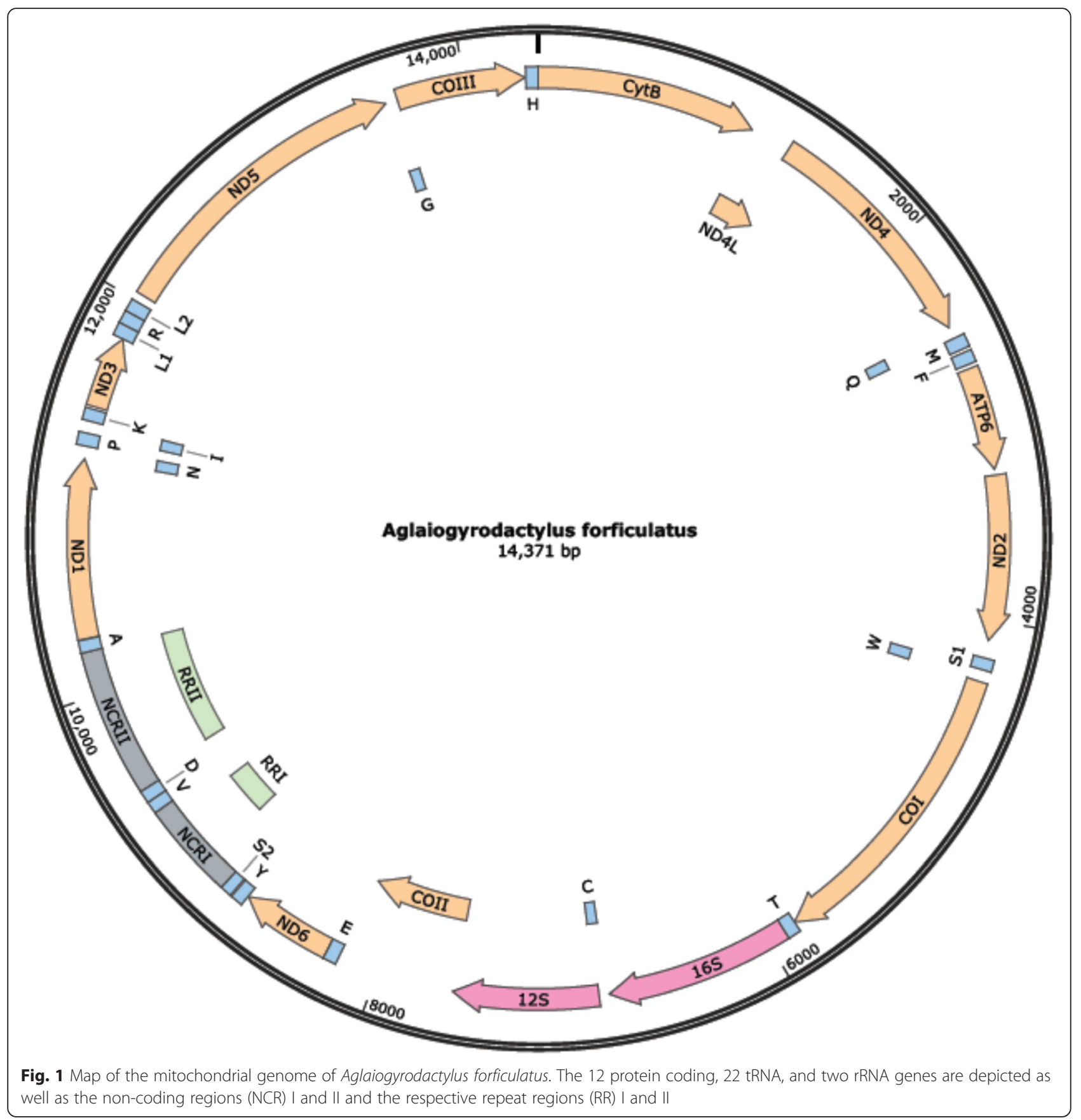

removed, the clade consisting of species of Gyrodactylidae received a bootstrap support of 100 .

The clustering of the mitochondrial genome of $A$. forficulatus with the remaining Gyrodactylidae species is nevertheless not strongly reflected in a matrix of pairwise genetic distances. Although the genetic distance between $A$. forficulatus and the closest relative $P$. variegatus was lowest (0.402; p-distances), the obtained values for all other pairwise comparisons were only slightly higher ranging from 0.409 with $N$. melleni to 0.477 with M. sebastis (Additional file 5). The respective values for the GBlock alignment were 0.396 for the comparison with $P$. variegatus and a range from 0.402 with $N$. melleni to 0.471 with $M$. sebastis (Additional file 5).

Non-coding regions (NCRs) including repeat regions I and II Two extended non-coding regions of $486 \mathrm{bp}$ and $733 \mathrm{bp}$, respectively, were detected in the mitochondrial genome of $A$. forficulatus. They are located relatively close to each other, separated only by the tRNA V and tRNA D genes, i.e. $123 \mathrm{bp}$, within a cluster of five tRNA genes that represents in comparison to other previously published 
Table 2 The mitochondrial genome of Aglaiogyrodactylus forficulatus; gene organization and gene order as determined by MITOS [8] and DOGMA [9]

\begin{tabular}{|c|c|c|c|c|}
\hline Gene & Position & Length (bp) & Start/Stop codon of PCGs & Best-fit model $^{c}$ \\
\hline Cytb & $1-1101$ & 1101 & ATG/TAG & $\mathrm{GTR}+\mathrm{G}$ \\
\hline ND4L & 1109-1363 & 255 & ATG/TAA & $\mathrm{GTR}+\mathrm{I}+\mathrm{G}$ \\
\hline ND4 & $1306-2508$ & 1203 & ATT/TAG & \\
\hline tRNA Q & $2504-2566$ & 63 & & $\mathrm{GTR}+\mathrm{I}+\mathrm{G}$ \\
\hline tRNA $M^{a}$ & $2568-2632$ & 65 & & $\mathrm{GTR}+\mathrm{I}+\mathrm{G}$ \\
\hline tRNA F $F^{a}$ & $2651-2713$ & 63 & & $\mathrm{GTR}+\mathrm{I}+\mathrm{G}$ \\
\hline ATP6 & $2735-3250$ & 516 & ATG/TAA & $\mathrm{GTR}+\mathrm{I}+\mathrm{G}$ \\
\hline ND2 & $3280-4104$ & 825 & $\mathrm{ATT} / \mathrm{TAG}$ & $\mathrm{GTR}+\mathrm{I}+\mathrm{G}$ \\
\hline tRNA S1 & $4193-4251$ & 59 & & $\mathrm{GTR}+\mathrm{I}+\mathrm{G}$ \\
\hline tRNA W & $4252-4314$ & 63 & & $\mathrm{GTR}+\mathrm{I}+\mathrm{G}$ \\
\hline $\mathrm{COI}$ & $4312-5841$ & 1530 & ATG/TAG & $\mathrm{GTR}+\mathrm{I}+\mathrm{G}$ \\
\hline tRNA $T^{\mathrm{a}}$ & $5842-5906$ & 65 & & $\mathrm{GTR}+\mathrm{I}+\mathrm{G}$ \\
\hline $16 S$ & $5907-6834$ & 928 & & $\mathrm{GTR}+\mathrm{I}+\mathrm{G}$ \\
\hline tRNA C & $6843-6901$ & 59 & & $\mathrm{GTR}+\mathrm{I}+\mathrm{G}$ \\
\hline 125 & $6887-7615$ & 729 & & $\mathrm{GTR}+\mathrm{I}+\mathrm{G}$ \\
\hline COII & $7613-8188$ & 576 & ATG/TAG & $\mathrm{GTR}+\mathrm{I}+\mathrm{G}$ \\
\hline tRNA E & $8205-8270$ & 66 & & $\mathrm{GTR}+\mathrm{I}+\mathrm{G}$ \\
\hline ND6 & $8272-8742$ & 471 & ATG/TAA & $\mathrm{GTR}+\mathrm{I}+\mathrm{G}$ \\
\hline tRNA Y & 8746-8808 & 63 & & $G T R+I+G$ \\
\hline tRNA S2 & $8817-8873$ & 57 & & $G T R+I+G$ \\
\hline Non-coding region I & 8874-9358 & 485 & & \\
\hline Repeat region I & $9017-9277$ & 261 & & \\
\hline tRNA V & $9359-9420$ & 62 & & $\mathrm{GTR}+\mathrm{I}+\mathrm{G}$ \\
\hline tRNA D ${ }^{a}$ & $9421-9482$ & 62 & & $\mathrm{GTR}+\mathrm{I}+\mathrm{G}$ \\
\hline Non-coding region II & $9483-10216$ & 733 & & \\
\hline Repeat region II & 9539-10212 & 675 & & \\
\hline tRNA $A^{a}$ & 10217-10277 & 61 & & $\mathrm{GTR}+\mathrm{I}+\mathrm{G}$ \\
\hline ND1 & 10282-11175 & 894 & ATG/TAG & \\
\hline tRNA N ${ }^{a}$ & $11175-11237$ & 63 & & $\mathrm{GTR}+\mathrm{I}+\mathrm{G}$ \\
\hline tRNA $P^{b}$ & 11238-11301 & 64 & & $\mathrm{GTR}+\mathrm{I}+\mathrm{G}$ \\
\hline tRNA I & 11300-11362 & 63 & & $\mathrm{GTR}+\mathrm{I}+\mathrm{G}$ \\
\hline tRNA Ka & $11363-11423$ & 61 & & $\mathrm{GTR}+\mathrm{I}+\mathrm{G}$ \\
\hline ND3 & 11438-11800 & 363 & ATG/TAG & $\mathrm{GTR}+\mathrm{G}$ \\
\hline tRNA $L 1^{a}$ & $11801-11865$ & 65 & & $\mathrm{GTR}+\mathrm{I}+\mathrm{G}$ \\
\hline tRNA R & 11866-11931 & 66 & & $\mathrm{GTR}+\mathrm{I}+\mathrm{G}$ \\
\hline tRNA L2 & 11932-11995 & 64 & & $\mathrm{GTR}+\mathrm{I}+\mathrm{G}$ \\
\hline ND5 & 12038-13597 & 1560 & ATG/TAA & $\mathrm{GTR}+\mathrm{I}+\mathrm{G}$ \\
\hline tRNA G & 13597-13658 & 62 & & $G T R+I+G$ \\
\hline COIII & 13659-14304 & 645 & ATG/TAA & $G T R+I+G$ \\
\hline tRNA H & 14308-14369 & 63 & & $\mathrm{GTR}+\mathrm{I}+\mathrm{G}$ \\
\hline
\end{tabular}




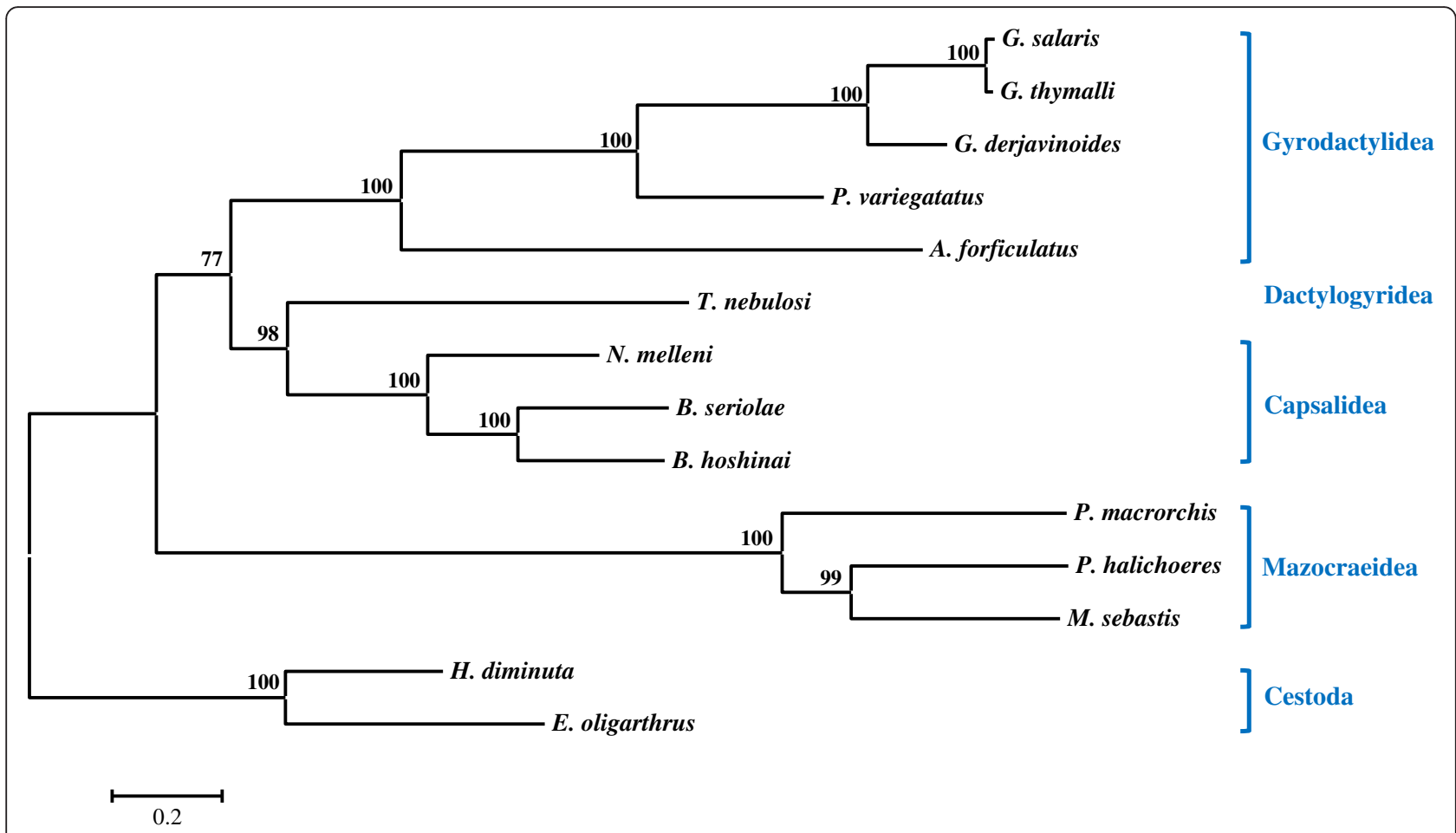

Fig. 2 Maximum Likelihood tree based on the concatenated MAFFT alignments of mitochondrial genes of A. forficulatus and 11 further Monogenoidea species. The cestode species $\mathrm{H}$. diminuta and E. oligarthrus served as outgroup. Bootstrap support values are indicated

monogenoidean mitochondrial genomes a substantially rearranged part of the mtDNA (Additional file 6). Both non-coding regions include a repetitive region consisting of three repeats. In repeat region I the repeats are $88 \mathrm{bp}$ long while they span 233 bp in repeat region II. For both repetitive sequence motifs blast searches revealed no significant sequence similarity to other GenBank entries. The average sequence similarity between the repeats was $81.1 \%$ in repeat region I and $96.5 \%$ in repeat region II, with somewhat higher sequence divergence in the beginning and the end of the repeated arrays (Additional file 7). The AT-content of repeat region I is $75.48 \%$, and repeat region II is particularly AT rich (83.38 \%) and may contain important functional domains for replication and transcription. However, such functional domains have not been described yet in monogenoidean flatworms in more detail than e.g. "Poly $\mathrm{T}$ stretch" or "A + T - rich segment" [13], and similar domains can be certainly found in the repeat region of $A$. forficulatus as well. Nevertheless, without a comprehensive functional analysis the identification of such functional domains would remain highly speculative.

The structure of the non-coding regions of $A$. forficulatus is strikingly similar to that of $P$. variegatus [13] although there is only one non-coding region in the latter species. The non-coding region in $P$. variegatus also consists essentially of two repetitive regions, one consisting of two 394 bp repeats (termed part I and
II), and one consisting of three $81 \mathrm{bp}$ repeats (termed part III). In contrast, the two non-coding regions observed in the mitochondrial genomes of the other included Gyroadactylidae species G. salaris [14], G. thymalli [15], and G. derjavinoides [16] do not consist of internal repeats. However, in these species the sequences of the two repeat regions are highly similar to each other indicating on the one hand that they originated from a duplication and on the other hand that they bear some functional domains.

\section{Gene order}

As proposed by the CREx program [30] P. variegatus and T. nebulosi (both species share identical mitochondrial gene orders except for the repetitive non-coding region in $P$. variegatus) have the highest similarity values in gene order based on inferred common intervals (Additional file 8). The program suggested a recombination scenario consisting of three transpositions and one complex tandem-duplication-random-loss (tdrl) event. The proposed transpositions (inversions; green boxes in Fig. 3) affect (a) tRNA-F and tRNA-M, (b) tRNA-A and tRNA-D, and (c) tRNA-R and tRNA-L2, and the proposed tdrl affect two larger gene blocks (red and blue boxes in Fig. 3). However, the proposed tdrl event can alternatively also be interpreted as a series of transpositions. Four larger gene blocks are conserved over all Gyrodactylidae species included in this comparison (Additional file 6). 


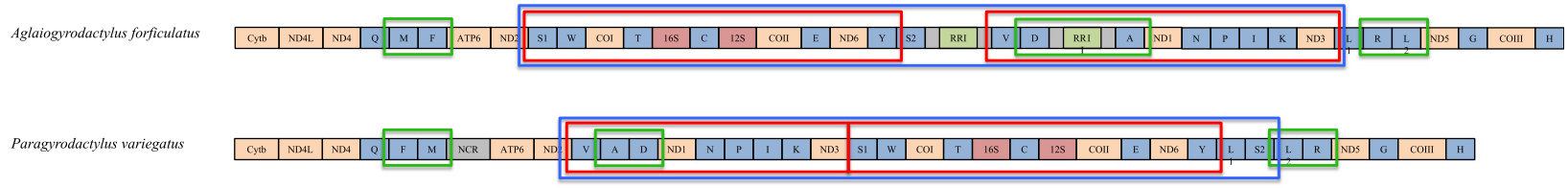

Fig. 3 Recombination scenario proposed by CREx [31] to explain mitochondrial gene order changes between A. forficulatus and $P$. variegatus. This includes three transpositions (green boxes) and one complex tandem-duplication-random-loss (tdrl) event (red and blue boxes)

Rearrangements of gene order have already been described for several mitochondrial genomes of Neodermata species (e.g. [13]). The four rearrangements in gene order of $A$. forficulatus in comparison to $P$. variegatus are thus not very surprising. As reported for other Monogenoidea mitochondrial gene order rearrangements mainly affect the specific position of some tRNA genes and non-coding regions. Also in A. forficulatus individual protein coding genes are not rearranged. For the mitochondrial genomes of the Gyrodactylidea species included in this study there are essentially four conserved regions; these are (i) ND5, tRNA G, COIII, tRNA H, Cytb, ND4L, ND4, (ii) ATP6, ND2, (iii) tRNA S1, tRNA W, COI, tRNA T, 16S, tRNA C, 12S, COII, tRNA E, ND6, tRNA Y, and (iv) ND1, tRNA N, tRNA P, tRNA I, tRNA K, ND3 (Additional file 6).

\section{Conclusions}

The mitochondrial genome of $A$. forficulatus shows a hitherto unique gene order within the monogenoiden Gyrodactylidea with four rearrangements in comparison to $P$. variegatus. The previously proposed sister group relationship of the oviparous and viviparous Gyrodactylidae is corroborated. However, more comprehensive sampling is required to further test the proposed phylogenetic hypothesis. All Gyrodactylidea mitochondrial genomes sequenced so far include repetitive regions, although the structure of two regions consisting of short tandemly arranged repeats that was found in the basal Gyrodactylidea lineages represented by $A$. forficulatus and $P$. variegatus differs substantially from the structure of two longer dispersed repeats in Gyrodactylus spp. The biological function of these repetitive regions is yet unknown but the sequencing of mitochondrial genomes of further Gyrodactylidae species may shed some light on the evolution of these regions.

\section{Additional files}

Additional file 1: Table S1. Base composition of the mtDNA. (DOCX $53 \mathrm{~kb}$ )

Additional file 2: Table S2. Codon usage of the protein coding genes (PCGs). (DOCX $107 \mathrm{~kb}$ )

Additional file 3: Figure S1. The secondary structures of the tRNAs. (PDF $184 \mathrm{~kb}$ )

Additional file 4: Figure S2. Maximum Likelihood trees after removal of ambiguous sites. (PDF $119 \mathrm{~kb}$ )
Additional file 5: Table S3. P-distances of concatenated mitochondrial genes. (DOCX $17 \mathrm{~kb}$ )

Additional file 6: Figure S3. Schematic order of the mitochondrial genes. (PDF $113 \mathrm{~kb}$ )

Additional file 7: Figure S4. Nucleotide sequence alignment of the repeat regions I and II. (DOCX $82 \mathrm{~kb}$ )

Additional file 8: Table S4. CREX distance matrix for mitochondrial gene order rearrangements. (DOCX $16 \mathrm{~kb}$ )

Competing interests

The authors declare that they have no competing interests.

\section{Authors' contributions}

$\angle B$ and $W A B$ designed the study. $L P A$ and WAB collected the parasite specimens. $\angle B$ performed the experimental work. $L B, B F$, and $W A B$ analyzed the data.

All authors co-drafted the manuscript and approved the final version.

\section{Acknowledgement}

We are grateful for having access to the Abel high computing facility of the University of Oslo (UiO) through the Norwegian Metacenter for Computational Science (NOTUR; grant k9201 nn) to LB. Article processing charges were kindly covered by the UiO publishing fund. Additional funding was provided from a grant of the Conselho de Desenvolvimento Científico e Tecnológico (CNPq), Brazil to WAB.

\section{Author details}

${ }^{1}$ Natural History Museum, University of Oslo, PO Box 1172, Blindern 0318, Oslo, Norway. ${ }^{2}$ Department of Tumor Biology, Institute for Cancer Research, Norwegian Radium, Hospital, Oslo University Hospital, PO Box 4950, Nydalen 0424, Oslo, Norway. ${ }^{3}$ Laboratório de Ecologia Molecular e Parasitologia Evolutiva-LEMPE, Universidade, Federal do Paraná-UFPR, Curitiba, Brazil.

Received: 17 February 2016 Accepted: 11 May 2016

Published online: 17 May 2016

\section{References}

1. Harris PD, Bachmann L, Bakke TA. The Parasites and Pathogens of the Atlantic Salmon: Lessons from Gyrodactylus salaris. In: Aas $\varnothing$, Einum S, Klemetsen A, Skurdal J, editors. Atlantic salmon ecology. Chichester (United Kingdom): Blackwell Publishing; 2011. p. 221-52.

2. Harris PD. The morphology and life cycle of the oviparous Oogyrodactylus farlowellae gen. et sp. n. (Monogenea, Gyrodactylidea). Parasitology. 1983; doi:10.1017/S0031182000082937.

3. Boeger WA, Kritsky DC, Belmont-Jégu E. Neotropical Monogenoidea. 20. Two new species of oviparous Gyrodactylidea (Polyonchoinea) from loricariid catfishes (Siluriformes) in Brazil and the phylogenetic status of Ooegyrodactylidae Harris, 1983. J Helminthol Soc Wash. 1994;61:34-44.

4. Boeger WA, Kritsky DC, Pie MR. Context of diversification of the viviparous Gyrodactylidae (Platyhelminthes, Monogenoidea). Zool Scr. 2003; doi:10.1046/j. 1463-6409.2003.00130.x.

5. Kritsky DC, Vianna RT, Boeger WA. Neotropical Monogenoidea. 50. Oviparous gyrodactylids from loricariid and pimelodid catfishes in Brazil, with the proposal of Phanerothecioides n. g., Onychogyrodactylus n. g. and Aglaiogyrodactylus n. g. (Polyonchoinea: Gyrodactylidea). Syst Parasitol. 2007; doi:10.1007/ s11230-006-9053-7. 
6. Hahn C, Bachmann L, Chevreux B. Reconstructing mitochondrial genomes directly from genomic next generation sequencing reads - a baiting and iterative mapping approach. Nucl Acids Res. 2013; doi:10.1093/nar/gkt371.

7. Bueno-Silva M, Boeger WA. Neotropical Monogenoidea. 58. Three new species of Gyrodactylus (Gyrodactylidae) from Scleromystax spp. (Callichthyidae) and the proposal of COll gene as an additional fragment for barcoding gyrodactylids. Folia Parasitol. 2014; doi:10.14411/fp.2014.028.

8. Bernt $M$, Donath $A$, Jühling $F$, Externbrink F, Florentz C, Fritzsch G, Pütz J, Middendorf M, Stadler PF. MITOS: Improved de novo Metazoan Mitochondrial Genome Annotation. Mol Phylogenet Evol. 2013; doi:10.1016/j.ympev.2012.08. 023. http://mitos.bioinf.uni-leipzig.de/index.py.

9. Wyman SK, Jansen RK, Boore JL. Automatic annotation of organellar genomes with DOGMA. Bioinformatics. 2004; doi:10.1093/bioinformatics. http://dogma. ccbb.utexas.edu/index.html.

10. Lowe TM, Eddy SR. tRNAscan-SE: A program for improved detection of transfer RNA genes in genomic sequence. Nucl Acids Res. 1997; doi:10. 1093/nar/25.5.0955. http://lowelab.ucsc.edu/tRNAscan-SE/.

11. Stothard P. The Sequence Manipulation Suite: JavaScript programs for analyzing and formatting protein and DNA sequences. Biotechniques. 2000;28:1102-4. http://www.bioinformatics.org/sms2/reference.html.

12. Noe L, Kucherov G. YASS: enhancing the sensitivity of DNA similarity search. Nucl Acids Res. 2005; doi: 10.1093/nar/gki478. http://bioinfo.lifl.fr/yass/yass.php.

13. Ye F, King SD, Cone DK, You P. The mitochondrial genome of Paragyrodactylus variegatus (Platyhelminthes: Monogenea): differences in major non-coding region and gene order to Gyrodactylus. Parasit Vectors. 2014; doi:10.1186/17563305-7-377.

14. Huyse T, Plaisance L, Webster BL, Mo TA, Bakke TA, Bachmann L, Littlewood DT. The mitochondrial genome of Gyrodactylus salaris (Platyhelminthes: Monogenea), a pathogen of Atlantic salmon (Salmo salar). Parasitology. 2007; doi:10.1017/S003118200600201.

15. Plaisance L, Huyse T, Littlewood DT, Bakke TA, Bachmann L. The complete mitochondrial DNA sequence of the monogenean Gyrodactylus thymalli (Platyhelminthes: Monogenea), a parasite of grayling (Thymallus thymallus). Mol Biochem Parasitol. 2007; doi:10.1016/j.molbiopara.2007.04.012.

16. Huyse T, Buchmann K, Littlewood DT. The mitochondrial genome of Gyrodactylus derjavinoides (Platyhelminthes: Monogenea) - a mitogenomic approach for Gyrodactylus species and strain identification. Gene. 2008; doi:10.1016/j.gene.2008.03.008.

17. Perkins EM, Donnellan SC, Bertozzi T, Whittington ID. Closing the mitochondrial circle on paraphyly of the Monogenea (Platyhelminthes) infers evolution in the diet of parasitic flatworms. Int J Parasitol. 2010; doi:10.1016/j.jpara.2010.02.017.

18. Kang S, Kim J, Lee J, Kim S, Min GS Park JK. The complete mitochondrial genome of an ectoparasitic monopisthocotylean fluke Benedenia hoshinai (Monogenea: Platyhelminthes), Mitochondrial DNA. 2012; doi: 10.3109/ 19401736.2012 .668900

19. Zhang J, Wu X, Li Y, Zhao M, Xie M, Li A. The complete mitochondrial genome of Neobenedenia melleni (Platyhelminthes: Monogenea): mitochondrial gene content, arrangement and composition compared with two Benedenia species. Mol Biol Rep. 2014; doi: 10.1007/s11033-014-3542-6.

20. Zhang J, Wu X, Li Y, Xie M, Li A. The complete mitochondrial genome of Tetrancistrum nebulosi (Monogenea: Ancyrocephalidae). Mitochondrial DNA. 2016; doi:10.3109/19401736.2013.867441.

21. Zhang J, Wu X, Xie M, Xu X, Li A. The mitochondrial genome of Polylabris halichoeres (Monogenea: Microcotylidae). Mitochondrial DNA. 2011; doi:10. 3109/19401736.2011.588223.

22. Park JK, Kim KH, Kang S, Kim W, Eom KS, Littlewood DT. A common origin of complex life cycles in parasitic flatworms: evidence from the complete mitochondrial genome of Microcotyle sebastis (Monogenea: Platyhelminthes). BMC Evol Biol. 2007; doi:10.1186/1471-2148-7-11.

23. Zhang J, Wu X, Xie M, Li A. The complete mitochondrial genome of Pseudochauhanea macrorchis (Monogenea: Chauhaneidae) revealed a highly repetitive region and a gene rearrangement hot spot in Polyopisthocotylea. Mol Biol Rep. 2012; doi:10.1007/s11033-012-1659-z.

24. Nakao M, McManus DP, Schantz PM, Craig PS, Ito A. A molecular phylogeny of the genus Echinococcus inferred from complete mitochondrial genomes. Parasitology. 2007; doi:10.1017/S003118200600193.

25. von Nickisch-Rosenegk M, Brown WM, Boore JL. Complete sequence of the mitochondrial genome of the tapeworm Hymenolepis diminuta: gene arrangements indicate that Platyhelminths are Eutrochozoans. Mol Biol Evol. 2001:18:721-30.
26. MAFFT version 7. Multiple alignment program for amino acid or nucleotide sequences. http://mafft.cbrc.jp/alignment/server/.

27. Castresana J. Selection of conserved blocks from multiple alignments for their use in phylogenetic analysis. Mol Biol Evol. 2000;17:540-52. http:// molevol.cmima.csic.es/castresana/Gblocks_server.html.

28. Sela I, Ashkenazy H, Katoh K, Pupko T. GUIDANCE2: accurate detection of unreliable alignment regions accounting for the uncertainty of multiple parameters. Nucl Acids Res. 2015; doi:10.1093/nar/gkq443. http://guidance. tau.ac.il/ver2/.

29. Lanfear R, Calcott B, Ho SWW, Guindon S. PartitionFinder: combined selection of partitioning schemes and substitution models for phylogenetic analyses. Mol Biol Evol. 2012; doi:10.1093/molbev/mss020

30. Guindon S, Dufayard JF, Lefort V, Anisimova M, Hordijk W, Gascuel O. New Algorithms and Methods to Estimate Maximum-Likelihood Phylogenies: Assessing the Performance of PhyML 3.0. Syst Biol. 2010; doi: 10.1093/ sysbio/syq010. http://www.atgc-montpellier.fr/phyml/.

31. Bernt M, Merkle D, Ramsch K, Fritzsch G, Perseke M, Bernhard D, Schlegel M, Stadler P, Middendorf M. CREx: Inferring Genomic Rearrangements Based on Common Intervals. Bioinformatics. 2007; doi:10.1093/bioinformatics/btm468.

32. Telford MJ, Herniou EA, Russell RB, Littlewood DT. Changes in mitochondrial genetic codes as phylogenetic characters: two examples from the flatworms. Proc Natl Acad Sci USA. 2000; doi: 10.1073/pnas.97.21.11359.

33. Wey-Fabrizius AR, Podsiadlowski L, Herlyn H, Hankeln T. Platyzoan mitochondrial genomes. Mol Phylogen Evol. 2013; doi:10.1016/j.ympev.2012.12.015.

34. Steinauer ML, Nickol BB, Broughton R, Orti G. First sequenced mitochondrial genome from the phylum Acanthocephala (Leptorhynchoides thecatus) and its phylogenetic position within Metazoa. J Mol Evol. 2005; doi:10.1007/ s00239-004-0159-8.

35. Fromm B, Burow S, Hahn C, Bachmann L. MicroRNA loci support conspecificity of the parasites Gyrodactylus salaris and G. thymalli (Platyhelminthes: Monogenea). Int J Parasitol. 2014; doi:10.1016/j.jpara.2014.05.010.

\section{Submit your next manuscript to BioMed Central and we will help you at every step:}

- We accept pre-submission inquiries

- Our selector tool helps you to find the most relevant journal

- We provide round the clock customer support

- Convenient online submission

- Thorough peer review

- Inclusion in PubMed and all major indexing services

- Maximum visibility for your research

Submit your manuscript at www.biomedcentral.com/submit
Ciomed Central 